\title{
TRATAMENTO COM ANTIDEPRESSIVOS TRICICLICOS AUMENTAM AS CHANCES DE DELIRIUM EM IDOSOS
}

Liege Camargo Alves Kurrle; Laboratório de Biogenômica, Universidade Federal de Santa Maria; liegekurrle@gmail.com;

Fernanda Barbisan; PPG Gerontologia, Universidade Federal de Santa Maria; fernandabarbisan@gmail.com;

Melissa Agostini Lampert; PPG Gerontologia, Universidade Federal de Santa Maria; melissa.a.lampert@gmail.com

Liana Pinheiro Santos Marques; PPG Gerontologia, Universidade Federal de Santa Maria; lips30@gmail.com Ivana Beatrice Mânica da Cruz; PPG Gerontologia, Universidade Federal de Santa Maria; ibmcruz@hotmail.com

Thamara Graziela Flores; PPG Farmacologia, Universidade Federal de Santa Maria; thamaraflores_fisio@yahoo.com.br

\section{RESUM0}

Introdução: Os antidepressivos tricíclicos (AT) são considerados padrão ouro de eficácia terapêutica frente a depressões graves, inclusive para idosos acima de 80 anos. Entretanto, os ATs apresentam maiores efeitos colaterais se comparados a outras classes, devido ao mecanismo de ação via bloqueio de recaptura de monoaminas, principalmente norepinefrina e serotonina, e em menor proporção dopamina. Em idosos ATs pioram o desempenho cognitivo, em superdosagens podem causar Delirium. Objetivo: Analisar associação entre o uso de ATs antes da internação em dosagens terapêuticas com o diagnóstico de Delirium. Método: A coleta de dados ocorreu nos anos 2015/2016 no Hospital Universitário de Santa Maria (RS). A amostra foi composta por 493 idosos, 89 idosos foram excluídos por não apresentarem dados suficientes. O diagnóstico de delirium na da admissão hospitalar foi obtido pelo Confusion Assessment Method(CAM). Análise estátistica via SPSS, $p<0,05$ considerado significativo. Resultados: O perfil da amostra com idade entre 60 e 69 anos $(48 \%)$, sendo $57,2 \%(\mathrm{n}=231)$ do sexo masculino, a prevalência de ingresso foi por fraturas gerais (20\%), neoplasias (19,6\%), e doenças cerebrovasculares (16,6\%). Houve associação entre o uso de ATs antes da admissão hospitalar e o diagnóstico de Delirium na admissão hospitalar $(p=0,035)$, com risco relativo para desenvolver o Delirium em 3,79 maior para os idosos que faziam uso de ATs. Conclusão: Mesmo quando administrado em doses terapêuticas os Ats, apresentaram associação com o diagnóstico de Delirium. Mais estudos precisam ser realizados para confirmação desta hipótese.

Palavras-chaves: Idoso; Delirium; Fármacos inapropriados. 